\title{
Monitoring of the thickness of the snow cover based on the neutron component data of cosmic rays
}

\author{
Kobelev $^{1}$ P.G., Dorman ${ }^{1,2 *}$ L.I., Trefilova ${ }^{1}$ L.A., Belov ${ }^{1}$ S.M., Pustilnik ${ }^{2}$ L.A., Zobnin ${ }^{1}$ \\ G.I., Yanke ${ }^{1}$ V.G. \\ ${ }^{I}$ Pushkov Institute of Terrestrial Magnetism, Ionosphere and Radiowave Propagation of the Russian \\ Academy of Sciences (IZMIRAN), Troitsk, Moscow, Russia \\ ${ }^{2}$ Israel Cosmic Ray and Space Weather Center with Emilio Segre Observatory on Mt. Hermon, affiliated \\ to Tel Aviv University, Ariel University, Shamir Research Institute, and Israel Space Agency, Israel \\ *Presenter author, e-mail: lid010529@gmail.com
}

OtherE-mails: kobelev@izmiran.ru,trefilova@izmiran.ru,sfrovis@gmail.com, levpust2149@gmail.com,egorizzee@gmail.com,yanke@izmiran.ru

\begin{abstract}
In the monograph of Lev Dorman on "Cosmic Rays in the Earth Atmosphere and Underground" [1a] were considered in Chapters 17 and 18 physical principles of many possible applications of the Cosmic Ray (CR) variations science to different aspects of technological, geological, meteorological, agricultural, archeological, mixing and exchanging of elements and isotopes between several planetary reservoirs, climate change, influence on Ecology Nuclear and Hydrogen bomb explosions and many other directions of historic and modern Earth Civilization Activity. Among agricultural CR application is an especially important monitoring the moisture content in the soil and snow above the soil using neutron detectors of a special design. is being actively developed.

At present, for this application is developed a special technology, which provides the ability to measure the moisture content in soil and snow above on some field scale in real-time. The described method is integral, i.e. averages the effect over the area determined by the range of neutrons in the lower atmosphere, which is $\sim 220 \mathrm{~m}$, and the corresponding area is about 10 hectares. For mountainous areas for northern countries, monitoring the thickness of the snow cover is especially relevant. The method used to estimate moisture and snow is a relative method and therefore requires calibration of detectors. For the winter period, such calibration has been carried out. The thickness of the snow cover during the period under consideration was insignificant at the beginning of the year and reached a maximum of $110 \mathrm{~mm}$ w.e., the error is estimated as $4 \mathrm{~mm}$ w.e.
\end{abstract}




\section{Introduction}

In recent years, it has been shown once again that, based on neutron detectors of cosmic rays $[1 \mathrm{~b}]$, it is possible to successfully implement promising methods for controlling soil moisture and the thickness of the snow cover over it. Subsequent work, however, is mainly devoted to the development of methods for assessing soil moisture and its dynamics. But for mid-latitude areas, which are covered by snow up to half of the time, monitoring the thickness of the snow cover is also important.

Various detectors have been developed to measure the dynamics of snow cover, which have their own strengths and limitations [2]. Conventional snow monitoring methods and tools provide local measurements. In addition, they almost always give the geometric thickness of the snow, and for practical purposes, it is important to know the thickness in units of the amount of substance or in units of water equivalent (hereinafter referred to as SWE or mm w.e.).

Non-destructive methods for determining the thickness of the snow cover are based on the absorption of a particular type of radiation and directly give the result in units of the amount of matter. In principle, it is possible to use three types of radiation with the different penetrating ability and allowing to measure different thicknesses of snow and two methods - the absorption method and the reflection method.

So for the method of absorption of radiation the maximum thickness of snow in the case of gamma radiation $[3,4] \sim 50 \mathrm{~mm}$ w.e., in the case of neutron radiation [5-7] $\sim 500 \mathrm{~mm}$ w.e., and in the case of muon radiation $\sim 3000 \mathrm{~mm}$ w.e.

For thermal and epithermal neutrons, to estimate the amount of a hydrogen-containing substance, it is possible not only to absorb radiation when the detector is under a layer of matter but also a reflection method when the detector is located above a layer of snow [8-13]. And the higher the detector is located, the more area the detector is scanning. The area is ultimately determined by the range of thermal and epithermal neutrons in air, which is $\sim 220 \mathrm{~m}$ and provides an area of 12-16 hectares[8, 13, 14, 15].

But the reflection method for estimating the thickness of the snow cover has several significant limitations. First, this method cannot distinguish the dynamics of snow thickness from the dynamics of soil moisture. Second, there is a limited range of snow depth that can be detected by neutron reflection. Based on simulations of neutron transport, it was found in [10] that the upper limit can be about $150 \mathrm{~mm}$ w.e. For mountainous heights, it has been found that the method works up to $600 \mathrm{~mm}$ w.e., since the count rate for these heights is about 2 pps [11]. In our case, such a high snow cover was not observed at sea level, and the count rate for an epithermal neutron detector was about 10 pps.

In the studies mentioned above, the conversion of neutron intensity data to snow depth is carried out using various approaches, including empirical linear regression functions [9], recalibration of the standard calibration function $\mathrm{N}_{0}$ [11], and a more physically sound approach in the work [10]. In [16], four different methods of converting the neutron count rate to the dynamics of snow cover were tested on the basis of long time series of measurements at a mountain range. 
The aim of this work is to study ground-based thermal and epithermal albedo neutrons to estimate the thickness of the snow cover in winter at constant soil moisture. The assessments will be carried out based on the monitoring data of the detectors of thermal and epithermal neutrons operating at the SKL Moscow. For calibration, the data of the weather station and direct measurements of the thickness of the snow cover will be used. For the autumn-spring period, a simplified accounting of the process of snow melting will be carried out on the basis of modeling the heat balance.

\section{The composition of the experimental complex}

The work was based on experimental data from the Mobile Cosmic Rays Laboratory (MCRL). The laboratory is equipped with three cosmic ray neutron detectors and a cubic muon telescope (Fig.1).

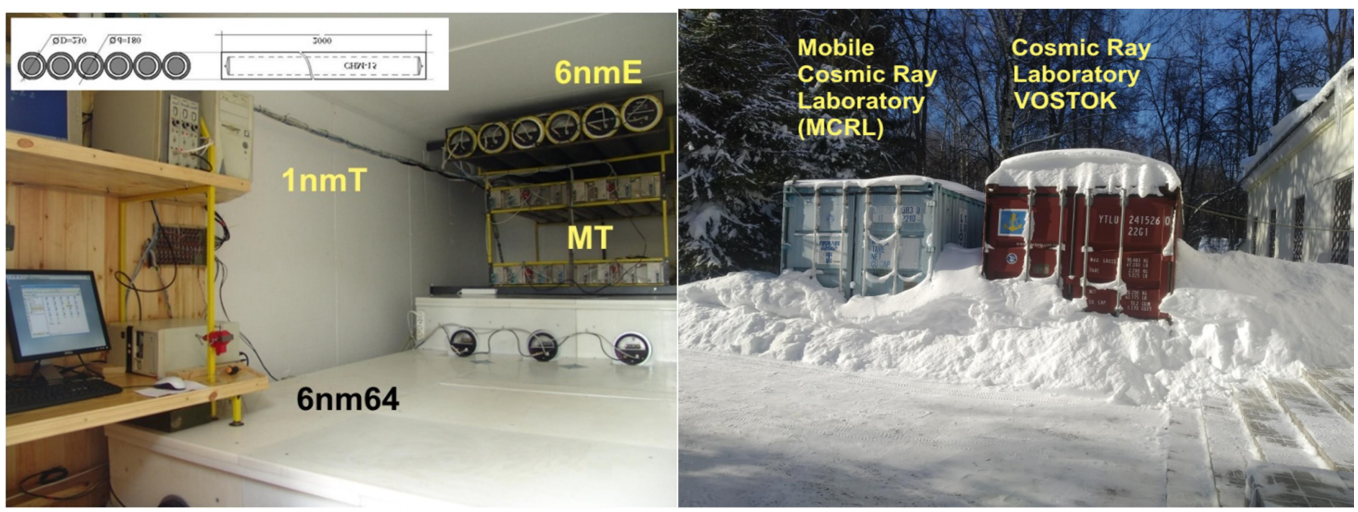

Fig.1. Standard neutron monitor (6nm64) and detectors of epithermal $(6 \mathrm{nmE})$ and thermal $(1 \mathrm{nmT}-$ not shown) neutrons in the MCRL.

The $6 \mathrm{~nm} 64$ standard neutron monitor is designed for monitoring cosmic rays of galactic origin and is only sensitive to particles $>20 \mathrm{MeV}$. The detector data is used as a reference to exclude primary variations from the observational data of other neutron detectors. The count rate of the section of a standard $6 \mathrm{~nm} 64$ neutron monitor is about 58 pps.

The $6 \mathrm{nmE}$ neutron detector of epithermal neutrons (Fig.1) is sensitive to neutrons with energies $>0.5 \mathrm{eV}$ and is based on $6 \mathrm{CHM}-15$ boron counters with a $2.5 \mathrm{~cm}$ neutron moderator. The epithermal neutron count rate is about 12 pps.

The $1 \mathrm{nmT}$ thermal neutron neutron detector (not shown in Fig.1) is sensitive to neutrons with energies $<0.5 \mathrm{eV}$ and is based on a single CHM-15 boron counter. The counting rate of thermal neutrons with such a detector is about $1.5 \mathrm{pps}$. In our case, the measurement errors of epithermal and thermal neutrons are about $0.5 \%$ and $1.3 \%$, respectively, for hourly averaging.

For automatic measurements of meteorological parameters necessary to correct a series of epithermal neutron data, to calibrate the detector, as well as to obtain moisture value, data from the Vaisala WXT530 automatic weather station are used.

The Vaisala ROSA automatic road weather station was used to determine the type of precipitation, because the data is measured using a laser that distinguishes the consistency of precipitation (rain / snow). 


\section{Data and their correction}

The neutron flux at the observation level is subject to primary variations in cosmic rays, changes associated with changes in atmospheric pressure, and changes in the content of water vapor in the air. To exclude such influence, it is needed to correct a measured count rate $N_{U}(t)$ by correction coefficients $N_{C}(t)=N_{U}(t) f_{P} f_{H} / f_{V}$ As a result, the corrected data $N_{C}(t)$ will contain only information about the moisture content in the soil and the thickness of the snow cover (Fig.2).

The barometric effect of the neutron component, the most significant and most easily taken into account, is defined as

$$
f_{P}(t)=\exp \left(-\beta\left(P-P_{0}\right)\right)
$$

where $P_{0}$ is an average pressure at the location of this detector, $\beta$ is the barometric coefficient which is $\beta=0.63 \% / \mathrm{mb}$ for epithermal neutrons and depends on the design of the detector[17].

Table 1. Count rate, barometric coefficient and coupling coefficient for various detectors.

\begin{tabular}{|l|l|l|l|}
\hline & $\mathbf{2 4 n m 6 4}$ & $\mathbf{6 n m E}$ & $\mathbf{1 n m T}$ \\
\hline $\mathbf{N}, \mathbf{H z}$ & 250 & 12 & 2 \\
\hline $\boldsymbol{\beta}, \mathbf{\%} / \mathbf{m b}$ & $0.74 \pm 0.01$ & $0.63 \pm 0.05$ & $0.61 \pm 0.07$ \\
\hline $\mathbf{C}$ n & 0.933 & 0.30 & 0.20
\end{tabular}

Variations in epithermal neutrons due to the barometric effect reach $10 \%$. Variations of galactic cosmic rays can be excluded from the data of the working detector using a correction factor as the ratio of the counting rate of the reference detector to the counting rate in the base period.

$$
f_{V}(t)=N^{S} / N_{\text {Base }}^{S}
$$

The base period of the reference detector must coincide with the base period of the main one. Ideally, the reference detector should also be an epithermal neutron detector, which would be in an environment free from the influence of the environment, which, however, is very difficult to achieve. Therefore, in practice, as a rule, a standard neutron monitor is used as a reference detector. This situation can be corrected by using the method of coupling coefficients [18]. Writing down (2) through variations

$$
f_{V}(t)=N^{S} / N_{\text {Base }}^{S}=v^{S}+1,
$$

where $\nu^{S}$ is the variations of galactic rays observed on a reference detector, which can be significantly different on a working detector with excellent geometry. The variations of these two detectors are related as

$$
v=\frac{C_{0}}{C_{0}^{S}} v^{S}
$$

where $C_{0}$ and $C_{0}^{S}$ are the coupling coefficients of main and reference detectors. Indeed, in the approximation of the zero harmonic, the variations for the main detector can be written as $v=a_{1} C_{0}$ and for the reference one as $v^{S}=a_{1} C_{0}^{S}$.

As a result, the correction factor for the correction for variations in galactic cosmic rays of the working detector is

$$
f_{V}(t)=\frac{C_{0}}{C_{0}^{S}} v^{S}+1
$$

In fact, expression (5) is an approximate expression. The exact solution can be obtained by the method of variations. Measured variation of the main detector $v=v_{E n v}+v_{G}$ can be corrected to exclude primary variations simply by subtracting last ones $v-v_{G}$. The variations $v_{G}$ determined by the GSM or some other approximate method. The primary variations 


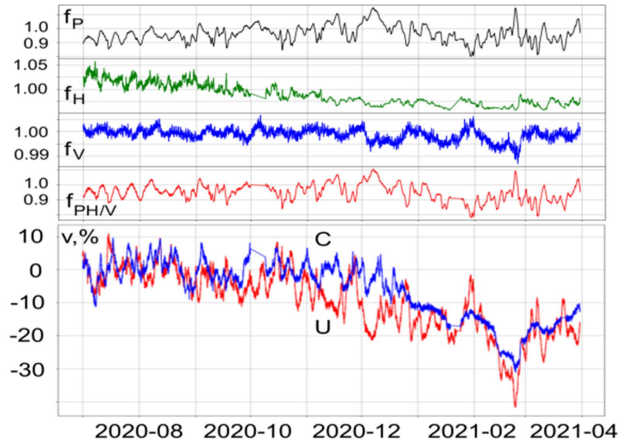

of epithermal neutrons can reach 5\%.

Changes in air humidity for epithermal neutrons for each point is defined as [1b]

$$
f_{H}(t)=1+0.0054 \cdot\left(\rho(t)-\rho_{20^{\circ} \mathrm{C} / 50 \%}\right),
$$

where $\rho(t)$ is an absolute air humidity at temperature $t, \rho_{20^{\circ} \mathrm{C} / 50 \%}=8.7 \mathrm{~g} / \mathrm{m}^{3}$ - absolute air humidity at standard circumstances $\left(20^{\circ} \mathrm{C}, 50 \%\right)$.

Fig.2. The time course of correcting coefficients for atmospheric pressure, air humidity and primary variations for epithermal neutrons and the final result. At bottom panel a comparison of the variations of the initial data of epithermal neutrons (red curve) and those corrected taking into account all distorting factors (blue curve) is shown.

Variations in epithermal neutrons caused by changes in air humidity can reach $\sim 5 \%$. The corrected counting rate of neutron detectors is converted to soil moisture and snow mass using the standard $\mathrm{N}_{0}$ calibration function proposed in [8] and reflecting the inverse relationship of the counting rate to the soil and above ground moisture content.

$$
\theta=\rho_{0} \frac{a_{0}}{\frac{N}{N_{0}}-a_{1}}-a_{2}, \quad \rho_{0} \frac{a_{0}}{(v+1)-a_{1}}-a_{2}
$$

where $\theta$ is the volumetric moisture content in the soil $\left(\mathrm{m}^{3} / \mathrm{m}^{3}\right), \rho_{0}$ is the dry matter density $(\mathrm{g} /$ $\left.\mathrm{cm}^{3}\right), N_{0}$ is the intensity of delayed neutrons over dry soil, which must be calibrated using the soil reference data in situ, and $\alpha_{\mathrm{i}}$ are the adjustable parameters $\left(a_{0}=0.0808, a_{1}=0.372, a_{2}=\right.$ $0.115)$. These parameters were for the total silica soil matrix.

\section{The ratio of thermal to epithermal neutrons}

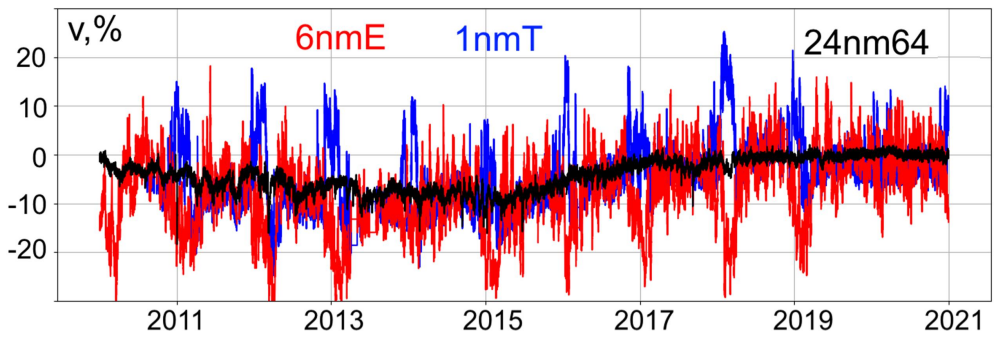

Fig.3. Long-term course of variations of epithermal $6 \mathrm{nmE}$ and thermal $1 \mathrm{nmT}$ neutrons and comparison with variations of a $24 \mathrm{~nm} 64$ neutron monitor. The base period is 2020-08.

Secondary neutron radiation is dominated by fast neutrons, which slow down to epithermal $(>0.5 \mathrm{eV})$ and thermal $(<0.5 \mathrm{eV})$ neutrons, when they propagate and interact with air nuclei and soil. It is obvious that the deceleration of epithermal neutrons is large in the soil and little in the air, since the water content in the soil is much higher. It was experimentally found that the decrease in the thermal neutron flux is proportional to the height above the earth's surface (that is, the main source of thermal neutrons). There are many hopes regarding the use of thermal neutrons: the use of the neutron energy spectrum to obtain information on the spatial 
structure of land surface waters, to estimate the amount of biomass, the use of the ratio of thermal and epithermal neutrons to distinguish snow from rain [8]. It was shown in the work that snow phenomena are usually characterized by a rapid increase in the intensity of thermal neutrons with the first centimeters of snow, followed by a rapid decrease, while the intensity of epithermal neutrons decreases monotonically with an increase in the depth of the snow cover. This conclusion is consistent with the results of neutron modeling of snow cover effects $[8,13]$ also found that changes in the spatial distribution of snow cover cause a hysteresis loop in the intensity of thermal and epithermal neutrons.

We have a long-term number of observations with good statistics. Fig.3 compares the long-term course of variations in epithermal $6 \mathrm{nmE}$, thermal $1 \mathrm{nmT}$ neutrons, and variations in a $24 \mathrm{~nm} 64$ neutron monitor. The behavior of thermal and epithermal neutrons is fundamentally different and in the winter period is determined by the amount of snowfall. For the 2021-2021 season, they could not see a convincing hysteresis loop, nor other effects characteristic of thermal neutron radiation that can be used to distinguish snow from rain.

\section{Discussion of results.}

As a result of adjustments and calibrations, we obtained final data reflecting only information about the thickness of the snow cover and the moisture content of the soil. For calibration, snow samples were taken in 5 measurements at different dates by the sounding method.

The final result of the analysis for the winter period from $11 / 18 / 2020$ to $03 / 24 / 2021$ is shown in Fig.4. The top panel in Fig.4 shows the precipitation according to the Vaisala ROSA and WXT530 weather stations, divided into liquid and solid precipitation phases, and the middle panel in Fig. 4 shows the time course of temperature.

Accumulated precipitation according to the weather station is shown in the lower panel in Fig.4 (dots). At positive temperatures and under the influence of solar radiation (two main heat flows), the snow melts and partially evaporates. Taking into account the simplified modeling of the heat balance [19], the time dependence of the remaining snow layer is also shown in the bottom panel of Fig.4 (green curve) along with several gravimetric control measurements of snow thickness (green stars).

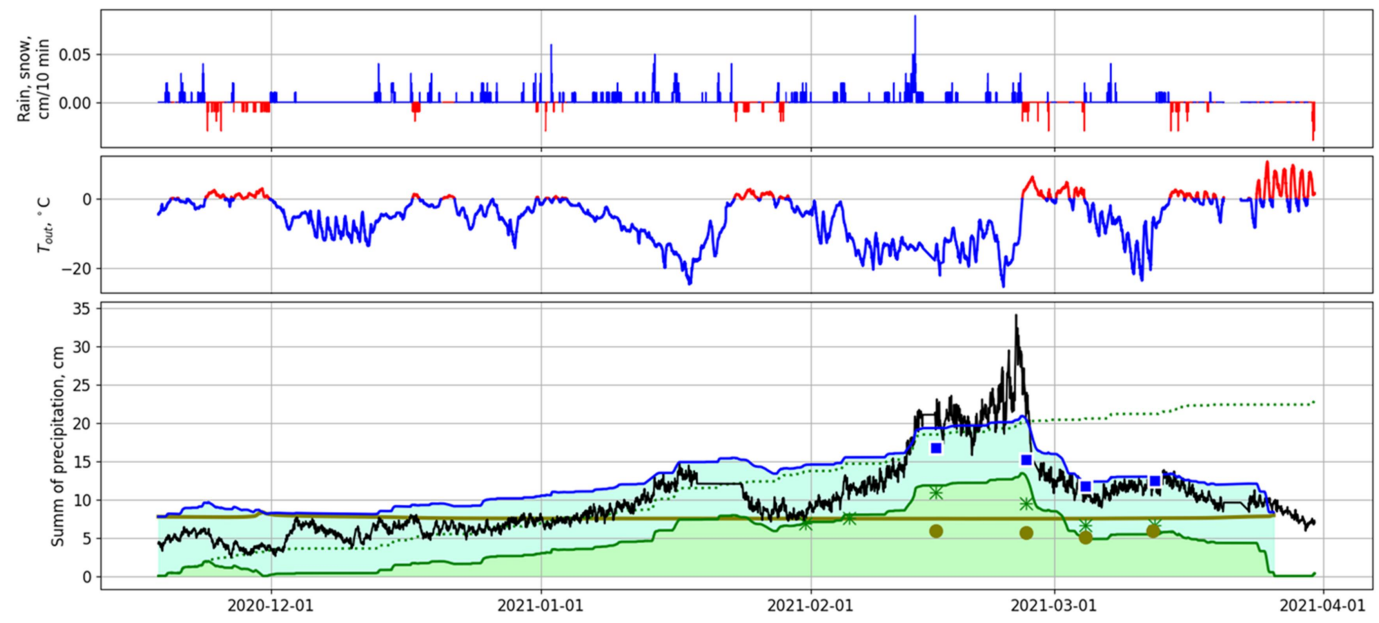

Fig.4. Top panel - precipitation in the form of snow (blue) or rain (red). Middle panel - air temperature. Bottom panel - result: Points curve

- precipitation according to the weather station data on a cumulative total, 
green curve and asterisks - real snow depth and points of calibration,

khaki line and mugs - moisture in the topsoil and points of calibration,

blue curve and squares - total quantity of moisture in and above the ground,

the black curve - is the total quantity of moisture in the soil and above the soil according to the

epithermal neutron detector data.

We tried to separate the problem of soil moisture and snow as much as possible, limiting ourselves to the winter period. During this period, the soil moisture practically does not change, which is reflected in Fig.4 (khaki line) along with several control gravimetric moisture measurements (circles). Experimentally, 5 soil samples were taken with a depth of $20 \mathrm{~cm}$. The soil moisture content of each measurement differed slightly and the average moisture content in the upper soil layer was equal to $55 \mathrm{~mm}$ we. The total amount of moisture (moisture in the soil and snow above the soil) is also given on the lower panels in Fig.4 (blue curve).

Finally, on the bottom panel of Fig.4 (black curve) shows the result of monitoring the thickness of the snow cover according to the epithermal neutron detector data. It is clear that the detector of epithermal neutrons with different weights is sensitive to all moisture, both in the soil and above it. The technique used by us for converting the detector count rate is described above. This result is compared with the result obtained by direct methods (blue curve). On the whole, there is a good agreement.

In the second mid-February, the data of the epithermal neutron detector record a sharp increase in the amount of snow. This is due to heavy snowfall, as a result of which a local accumulation of snow occurred over the detector, which gave a distortion in the data.

\section{Conclusions.}

The paper presents the result of continuous monitoring of the thickness of the snow cover in the very snowy winter period of 2020-2021 using an epithermal neutron detector operating at IZMIRAN since 2010. The result of monitoring the epithermal neutron detector for the period under study was compared with the direct data of precipitation from the meteorological station and sampling.

The results obtained confirm the correctness of the technique used, as well as the temporal stability of the detectors of epithermal and thermal neutrons.

The described method is integral, i.e. averages the effect on the area, determined by the range of neutrons in the lower atmosphere about $\sim 220 \mathrm{~m}$, and approximately equal to 10 hectares. Accuracy of estimates of snow cover thickness with hourly resolution $\Delta= \pm 4$ (stat.) \pm 20 (syst.) mm w.e.

The results are a good starting point for discussion and further research, which should include a closer look at the role of thermal neutrons in winter and summer. It is important to carry out Geant 4 simulations to select the type and design of the detector, select the optimal height for its location, determine the contribution of peripheral regions to the observed count rate, and for physical understanding and construction of more adequate models.

This work was performed on equipment at Russia's unique National Ground-Based Network of Cosmic Ray Stations.

\section{References}

[1a] Dorman L.I. (2004). Cosmic Rays in the Earth Atmosphere and Underground, Kluwer Academic Publishers, Dordrecht/Boston/London, pp XXXII +855.

[1b] Zreda, M., W. J. Shuttleworth, X. Xeng, C. Zweck, D. Desilets, T. E. Franz, R. Rosolem, and P. A. Ferre (2012), COSMOS: The Cosmic-ray Soil Moisture Observing System, Hydrol. Earth Syst. Sci. 
Discuss., 9, 4505- 4551, doi:10.5194/hessd-9-4505-2012.

[2] Pirazzini, R., Leppänen, L., Picard, G., Lopez-moreno, J. I., Marty, C., Macelloni, G., et al. (2018). European in-situ snow measurements: practices and purposes. Sensors 18:2016. doi: $10.3390 / \mathrm{s} 18072016$

[3] Peck E.L., Bisell V.C., Jones E.B., Burge D.L., Evaluation of snow water equivalent by airborne measurement of passive terrestrial gamma radiation. J. Hydrol. 7, 1151-1159, 1971.

[4] Choquette, Y., Ducharme, P., and Rogoza, J. (2013). "CS725, an accurate sensor for the snow water equivalent and soil moisture measurements" in Proceedings of the International Snow Science Workshop (Grenoble), 931-936.

[5] Kodama, M., Nakai, K., Kawasaki, S., and Wada, M. (1979). An application of cosmic-ray neutron measurements to the determination of the snow-water equivalent. J. Hydrol. 41, 85-92. doi: 10.1016/0022-1694(79)90107-0

[6] Kodama, M. (1980). Continuous monitoring of snow water equivalent using cosmic-ray neutrons. Cold Reg. Sci. Technol. 3, 295-303. doi: 10.1016/0165-232X(80)90036-1

[7] Gugerli, R., Salzmann, N., Huss, M., and Desilets, D. (2019). Continuous and autonomous snow water equivalent measurements by a cosmic ray sensor on an alpine glacier. Cryosphere 13, 34133434. doi: 10.5194/tc-13-3413-2019

[8] Desilets, D., M. Zreda, and T. P. A. Ferre (2010), Nature's neutron probe: Land surface hydrology at an elusive scale with cosmic rays, Water Resour. Res., 46, W11505, $\underline{2010}$ doi:10.1029/2009WR008726.

[9] Sigouin, M. J. P., and Si, B. C. (2016). Calibration of a non-invasive cosmic-ray probe for wide area snow water equivalent measurement. Cryosphere 10, 1181-1190. doi: 10.5194/tc-10-1181-2016

[10] Desilets, D. (2017). Calibrating a Non-invasive Cosmic Ray Soil Moisture Probe for Snow Water Equivalent. Hydroinnova Technical Document, 17-01.

[11] Schattan, P., Baroni, G., Oswald, S. E., Schober, J., Fey, C., Kormann, C., et al. (2017). Continuous monitoring of snowpack dynamics in alpine terrain by aboveground neutron sensing. Water Resour. Res. 53, 3615-3634. doi: 10.1002/2016WR020234

[12] Rivera Villarreyes et al., 2011 C.A. Rivera Villarreyes, G. Baroni, S.E. Oswald Integral quantification of seasonal soil moisture changes in farmland by cosmic ray neutrons Hydrol. Earth Syst. Sci., 15 (12) (2011), pp. 3843-3859 CrossRefView Record in ScopusGoogle Scholar

[13] Zweck, C., Zreda, M., and Desilets, D. (2013). Snow shielding factors for cosmogenic nuclide dating inferred from monte carlo neutron transport simulations. Earth Planet. Sci. Lett. 379, 64-71. doi: 10.1016/j.eps1.2013.07.023

[14] Kohli, M., M. Schron, M. Zreda, U. Schmidt, P. Dietrich, S. Zacharias, Footprint characteristics revised for field-scalesoil moisture monitoring with cosmic-ray neutrons, Water Resour. Res., 51,5772-5790, 2015, doi:10.1002/2015WR017169.

[15] Schron, M., Rosolem, R., Kohli, M.,Piussi, L., Schroter, I., Iwema, J., et al.(2018). Cosmic-ray neutron roversurveys of field soil moisture and theinfluence of roads. Water Resources Research, 54, 6441-6459. https://doi.org/10.1029/2017WR021719

[16] Bogena Heye R., Herrmann Frank, Jannis Jakobi, Cosimo Brogi, Andreas Ilias, Johan Alexander Huisman, Andreas Panagopoulos and Vassilios Pisinaras Monitoring of Snowpack Dynamics With Cosmic-Ray Neutron Probes: A Comparison of Four Conversion Methods Front. Water, 04 August 2020 https://doi.org/10.3389/frwa.2020.00019

[17] Kobelev P., Belov A., Mavromichalaki E., Gerontidou M., Yanke V. Variations of Barometric Coefficients of the Neutron Component in the 22-23 Cycles of Solar Activity Proc. 32 ${ }^{\text {nd }}$ (ICRC): Proc. Beijing. 2011. 4 p.

[18] Kobelev P., Belov A., Eroshenko E., Yanke V. Reception coefficients and energy characteristics of the ground level cosmic ray detectors. Proc. 33rd ICRC, Rio de Janeiro. 2013. id 0878. URL: $\mathrm{ftp}$ //cr0.izmiran.ru/Proceedings/(ICRC)

[19] Gordeev I.N. Methodology for calculating the intensity of snow melting in the forecasts of the spring 